\title{
Cardiac toxicity with monoclonal antibodies therapy
}

\author{
Haitham Mazek MD, Rashmi Verma MD, Jason Wischmeyer MD
}

\section{AbStRACT}

Breast cancers which overexpress the human epidermal growth factor receptor 2 (HER2) proteins generally have poor prognosis. Trastuzumab is a monoclonal antibody which inhibits HER-2 activation and has been shown to be an effective therapy for women with breast cancers that overexpress HER2 proteins. The most important side effect of trastuzumab is cardiotoxicity. We report a patient with left ventricular dysfunction and pericardial effusion after treatment with pertuzumab and trastuzumab.

Key words: cancer therapy, monoclonal antibodies, cardiac toxicity, pericardial effusion

\section{INTRODUCTION}

Pericardial effusion is an excessive accumulation of fluid in the pericardial space and can occur as a complication of malignancy and/or anticancer therapy. We report a patient who developed a nonmalignant pericardial effusion and left ventricular dysfunction after receiving treatment with the combination of pertuzumab and trastuzumab.

\section{CASE}

A 36-year-old woman with metastatic estrogen receptor, progesterone receptor, and HER2 positive invasive ductal carcinoma of the right breast was admitted to our hospital in June 2014 with severe dyspnea. The patient was originally diagnosed 18 months prior to admission with axillary lymph node and osseous metastasis. She received four cycles of weekly

Corresponding author: Haitham Mazek MD Contact Information: Haitham.mazek@ttuhsc.edu DOI: $10.12746 /$ swrccc2015.0311.148 gemcitabine, paclitaxel, and trastuzumab with monthly denosumab treatment and had a complete response. The patient continued on maintenance tamoxifen, trastuzumab, and denosumab after confirming a normal left ventricular ejection fraction (LVEF) of $60-64 \%$

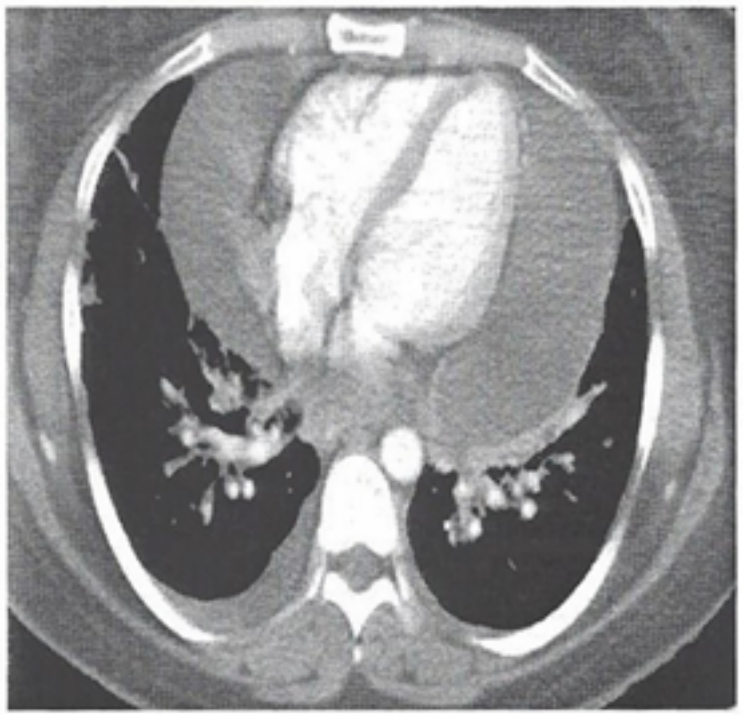

Figure1: Computed tomography of the thorax shows a large pericardial effusion. 
with no pericardial effusion. In March 2014 she presented with generalized lymphadenopathy indicative of progressive disease based on physical examination and computed tomography of her chest. She refused cytotoxic therapy, and pertuzumab was added to her trastuzumab regimen. During this admission (June 2014), she presented with severe dyspnea, and an echocardiogram confirmed the presence of a large pericardial effusion and a LVEF of $40 \%$. Trastuzumab was held. The patient underwent pericardiotomy with marked clinical improvement. Pericardial fluid was negative for malignant cells. Repeat echocardiogram in December 2014 showed a LVEF of $60 \%$.

\section{Discussion}

Pericardial effusion is a consequence of inflammation of the pericardium (pericarditis) secondary to infectious agents, inflammatory disorders, metastatic malignancies, uremia, congestive heart failure, and drugs, such as the antineoplastic agents doxorubicin, cyclophosphamide, and trastuzumab. Pertuzumab is a monoclonal antibody that selectively binds to the human epidermal growth factor receptor-2 protein
(HER-2) and inhibits its dimerization and subsequent activation. ${ }^{1,2}$ Pertuzumab has been approved by the FDA in combination with trastuzumab (a monoclonal antibody that also inhibits HER-2 activation) and docetaxel as first-line treatment for metastatic HER2-expressing breast cancer. ${ }^{1}$ A major complication of trastuzumab is cardiac dysfunction. The mechanism responsible for this cardiac complication is unknown and may be secondary to a sequential stress mechanism. Evidence from both in vivo and in vitro studies indicates the importance of the epidermal growth factor signaling system (HER2 or ErbB2) in the normal heart and suggests that trastuzumab cardiotoxicity is directly related to HER2 blockade. Although the role of HER2 in the pathophysiology of heart failure is not well understood, serum HER2 levels are increased in patients with chronic heart failure, and these levels correlate inversely with left ventricular function. ${ }^{3}$ Since HER-2 is involved in the growth and survival of adult cardiomyocytes, treatment with trastuzumab may result in a reversible decrease in LVEF in up to $20 \%$ of patients. ${ }^{4}$ This requires monitoring of LVEF during treatment, but the best schedule for LVEF monitoring in asymptomatic patients during trastuzumab treatment is uncertain. Most of the adjuvant trials have as-

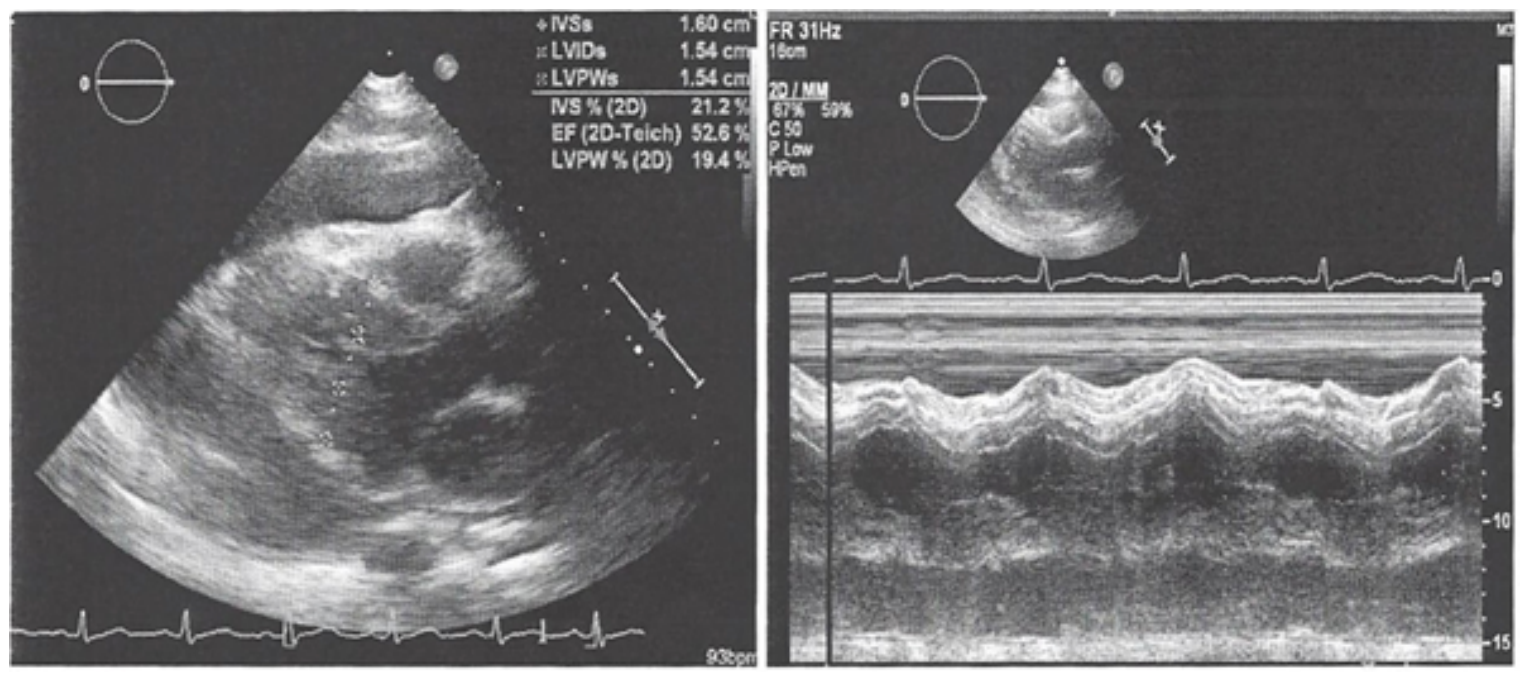

Figure2: Transthoracic echocardiography shows a pericardial effusion. 
sessed LVEF either by echocardiography or MUGA scans every three months up to the ninth month of treatment with a repeat assessment six months after the cessation of treatment. Based on these criteria, it was recommended to monitor cardiac function at three month intervals during trastuzumab treatment and every six months for at least two years after completion of treatment. Therapy should be held in patients who develop cardiac symptoms or a greater than $10 \%$ absolute asymptomatic decline in LVEF. Medical therapy should be initiated in patients with symptomatic heart failure. Improvement in cardiac function occurred in the majority of these patients. Our patient's new onset pericardial effusion and decline in LVEF developed only after pertuzumab was added to trastuzumab, suggesting that the combined anti-HER-2 regimen was responsible for this complication. Thus, our case report illustrates the importance of close follow-up and monitoring of patients receiving dual anti-HER-2 therapy for the early detection of this potentially life threatening complication.

\section{REFERENCES}

1. Jones AL, et al. Management of cardiac health in trastuzumab-treated patients with breast cancer: updated United Kingdom National Cancer Research Institute recommendations for monitoring. Br J Cancer 2009; 100: 684-692.

2. Agus DB, Akita RW, Fox WD, et al. Targeting ligand-activated ErbB2 signaling inhibits breast and prostate tumor growth. Cancer Cell 2002; 2: 127-137.

3. Perik PJ, de Vries EG, Gietema JA, et al. Serum HER2 levels are increased in patients with chronic heart failure. Eur J Heart Fail 2007; 9:173.

4. Baselga J, Swain SM. Novel anticancer targets: revisiting HER2 and discovering HER3. Nat Rev Cancer 2009; 9: 463-475. 5. Mackey JR et al. Cardiac management during adjuvant trastuzumab therapy: recommendations of the Canadian Trastuzumab Working Group. Curr Oncol 2008 Jan; 15(1): 24-35.
Author Affiliation: Haitham Mazek is a resident in Internal Medicine at Texas Tech University Health Sciences Center in Lubbock, TX. Rashmi Verma is a fellow in HematologyOncology in Internal Medicine at TTUHSC in Lubbock, TX. Jason Wischmeyer is a faculty member in Cardiology in Internal Medicine at TTUHSC in Lubbock, TX.

Received: 06/26/2015

Accepted: 07/13/2015

Reviewers: Catherine Jones MD

Published electronically: 07/15/2015

Conflict of Interest Disclosures: none 